\title{
Maturation of ovarian function in female ferrets is influenced by photoperiod
}

\author{
K. D. Ryan \\ Department of Physiology, University of Pittsburgh School of Medicine, Pittsburgh, \\ PA 15261, U.S.A.
}

\begin{abstract}
Summary. Sexual maturation of immature ferrets in response to a stimulatory photoperiod generally occurs within 6-7 weeks after imposition of long days at 16 weeks of age. In this study, females were examined for evidence of ovarian maturation after 22 days of exposure to the stimulatory photoperiod, before any sign of sexual maturation. The photoperiod-stimulated and control females were the same age (19 weeks), had similar body weights, and showed no evidence of vulvar oedema. Comparison of oestradiol secretory response to hCG stimulation and of follicular development in the ovary revealed marked ovarian stimulation in females kept in long days compared to control females kept in short days. A prolonged period of ovarian stimulation therefore occurs during exposure to long days before there is external evidence of sexual maturation in ferrets.
\end{abstract}

\section{Introduction}

The female ferret is a reflexly ovulating mammal in which sexual maturation can be accelerated by a stimulatory long-day photoperiod ( $16 \mathrm{~h}$ light : $8 \mathrm{~h}$ dark) imposed after 15 weeks of age (Thorpe, 1967). The external sign of sexual maturation, vulvar oedema, occurs about 6 weeks after onset of the stimulatory photoperiod (Thorpe, 1967; Donovan, 1967). However, ovarian follicular growth occurs before observable vulvar oedema in adults undergoing the seasonal onset of reproductive function (see Table 1, Hammond \& Marshall, 1930). More detailed studies of the process of sexual maturation in response to a long-day photoperiod have revealed that a decrease in efficacy of oestradiol negative feedback on LH secretion (Ryan, 1984), and a rise in pulsatile secretion of LH (Ryan \& Robinson, 1985), occur at or before the onset of pubertal vulvar oedema in developing ferrets. Ferrets can also secrete oestradiol in response to PMSG by the age of 12-15 weeks (Chang, $1950 ;$ K. D. Ryan, unpublished), indicating FSH receptors in the ovaries of immature ferrets at the age at which the puberty inducing stimulus was applied. In the following report, ovarian function and morphology of ferrets after exposure to long days for only 22 days are contrasted with those of controls kept in short days. These observations were thus made before the occurrence of other observed endocrine responses to the stimulus of a long-day photoperiod.

\section{Materials and Methods}

The ferrets used in this study were all obtained from Marshall Farms USA, North Rose, NY 14516, at 8 weeks of age. These females were provided with Purina Cat Chow and water ad libitum, and were housed, 3 per cage, in a windowless room with an $8 \mathrm{~h}$ light : $16 \mathrm{~h}$ dark cycle. Sexual maturation was induced by exposure of females to a long-day light cycle (16L:8D) beginning at 16 weeks of age, and studies were begun when females were 19 weeks of age. Control females remained in the non-stimulatory photoperiod. Lights came on in both rooms at $07: 00 \mathrm{~h}$. 
Experiment 1 . In this study, 10 females in long days and 10 in short days were fitted with jugular venous catheters to permit non-traumatic blood sampling. Catheters were inserted into the external jugular vein under pentobarbitone sodium anaesthesia $(45 \mathrm{mg} / \mathrm{kg})$ (Nembutal: Abbott Laboratories, North Chicago, IL 60064). The intravascular portion of the catheter was a 7-cm length of polyvinyl tubing $($ i.d. $=0.03$ in $(0.76 \mathrm{~mm}))$; this was joined by a solvent bond to a larger tube $($ i.d. $=$ 0.04 in $(1.02 \mathrm{~mm})$ ) for the external portion. The extravascular portion of the catheter was passed beneath the skin to emerge at the top of the head and was closed with a metal plug. The external portion of the catheter was not protected, and no damage by cage-mates occurred. Daily blood samples $(1.0 \mathrm{ml})$ were obtained in heparinized syringes from each female for 4 days. On the 5 th day and for 9 days thereafter, females received an i.m. injection of $0.25 \mathrm{ml}$ sterile saline $(0.9 \% \mathrm{w} / \mathrm{v}$ $\mathrm{NaCl}$ ) or $0.25 \mathrm{ml}$ sterile saline containing 5 i.u. hCG (Pregnyl; Organon, W. Orange, NJ). Females were examined daily for evidence of vulvar oedema.

Experiment 2. This study was performed to assess the morphology of ovaries from females at 19 weeks of age. Animals ( $N=5$, Group $S$ ) housed in short days and those which had been moved to long days at 16 weeks $(N=5$, Group $\mathrm{L})$ were ovariectomized under pentobarbitone sodium anaesthesia at 19 weeks of age. Additionally, smaller groups of females received either hCG ( 3 in Group L and 3 in Group S) or saline ( 2 in Group L and 3 in Group S) for $48 \mathrm{~h}$. The ovaries were then removed, immersed in Bouin's fixative, and embedded in paraffin wax. One ovary from each female was serially sectioned and stained with haematoxylin and eosin. Antral follicles were followed by light microscopy to the section containing the nucleolus of the ovum, and the area of that section of the follicle was measured by a computerized digitizing morphometry program (Bioquant II, R \& M Biometrics, 3325 Charlotte Avenue, Nashville, TN 37209).

Plasma oestradiol concentrations were measured by a radioimmunoassay employing an antiserum generously provided by Dr S. G. Hillier. This antiserum was produced in rabbits against $17 \beta$-oestradiol-6-bovine serum albumin and was used at a final dilution of $1: 100000$. The crossreactivity of the antiserum was that described by Scaramuzzi \& Land (1978): 100\% with oestradiol$17 \beta, 0.4 \%$ with oestradiol- $17 \alpha, 1.4 \%$ with oestrone and $0.5 \%$ with oestriol. Plasma samples were extracted with benzene and oestradiol was purified via Sephadex column chromatography. Recoveries determined by co-chromatography with $\left[{ }^{3} \mathrm{H}\right]$ oestradiol- $17 \alpha$ were $81 \cdot 0 \pm 1 \cdot 1 \%$. Interassay coefficients of variation were 16 at $20 \%$ bound $(25 \mathrm{pg} /$ tube $)$ and 21 at $88 \%$ bound $(0.9$ $\mathrm{pg} /$ tube). Assay of $0.50 \mathrm{ml}$ plasma from hypophysectomized ferrets gave a value of $2.5 \pm 0.2 \mathrm{pg}$ oestradiol $/ \mathrm{ml}$. Analysis of plasma profiles of oestradiol concentrations were performed by one-way analysis of variance for repeated measures (Zar, 1974).

\section{Results}

\section{Experiment 1}

In response to the hCG injections, plasma oestradiol concentrations rose more rapidly and to a higher level in females housed in long days for three weeks than in control females $(P<0.05)$ (Textfig. 1). The difference in response to hCG stimulation occurred in females that had similar basal steroid levels, and the rapid rise in plasma oestradiol concentrations did not occur in females treated only with saline (Text-fig. 1). Ferrets of this age and stage of development have not yet begun to show divergent body weights from the onset of oestradiol secretion (Hammond, 1974). At the end of this study, both groups of females had attained full vulvar oedema and even females in short days had plasma concentrations of oestradiol that were normal for oestrous females. The concentrations in females in long days after 6-9 days of hCG treatment were considerably higher than values typical of oestrus (K. D. Ryan, unpublished). After 9 days, the ovaries of both groups of females were maximally stimulated, so it was decided that more useful information would result from examining ovaries at the time the treatment was started when different responses to hCG were still apparent. 


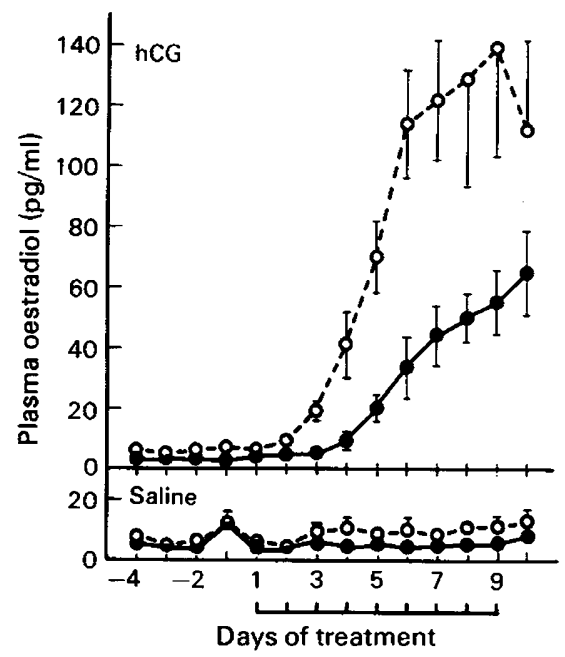

Text-fig. 1. Oestradiol secretory response of immature female ferrets at 19 weeks of age to daily injections of 5 i.u. hCG ( $\mathrm{N}=6$ for each group) or $0.25 \mathrm{ml}$ saline $(\mathrm{N}=4$ for each group). Injections were begun on Day 1 . Open circles represent data from females housed in long days (16L:8D) for 22 days; closed circles represent data from females housed continuously in short days $(8 \mathrm{~L}: 16 \mathrm{D})$. Where no standard error bars are shown, the error is covered by the symbol for the mean.

\section{Experiment 2}

There were clear differences in ovarian morphology between animals kept in short days (Group S) and after 3 weeks of long days (Group L). Group L females all exhibited several follicles of diameters $>0.6 \mathrm{~mm}$. In contrast, no follicles larger than $0.5 \mathrm{~mm}$ diameter were found in Group $S$ females (Text-fig. 2). Treatment of females with hCG for $48 \mathrm{~h}$ did not alter the profile of antral follicle development in females in either group (Text-fig. 3).

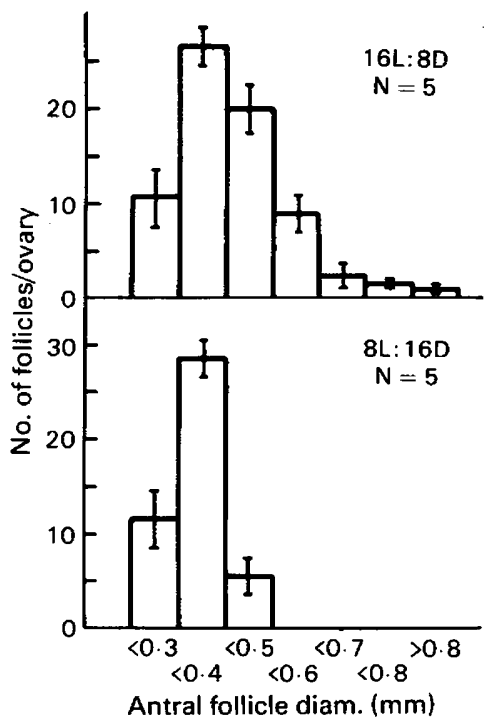

Text-fig. 2. Summary of morphometric analysis of ovaries from 19-week-old female ferrets kept in long or short days after 16 weeks of age ( 1 ovary of each of 5 females/group). 


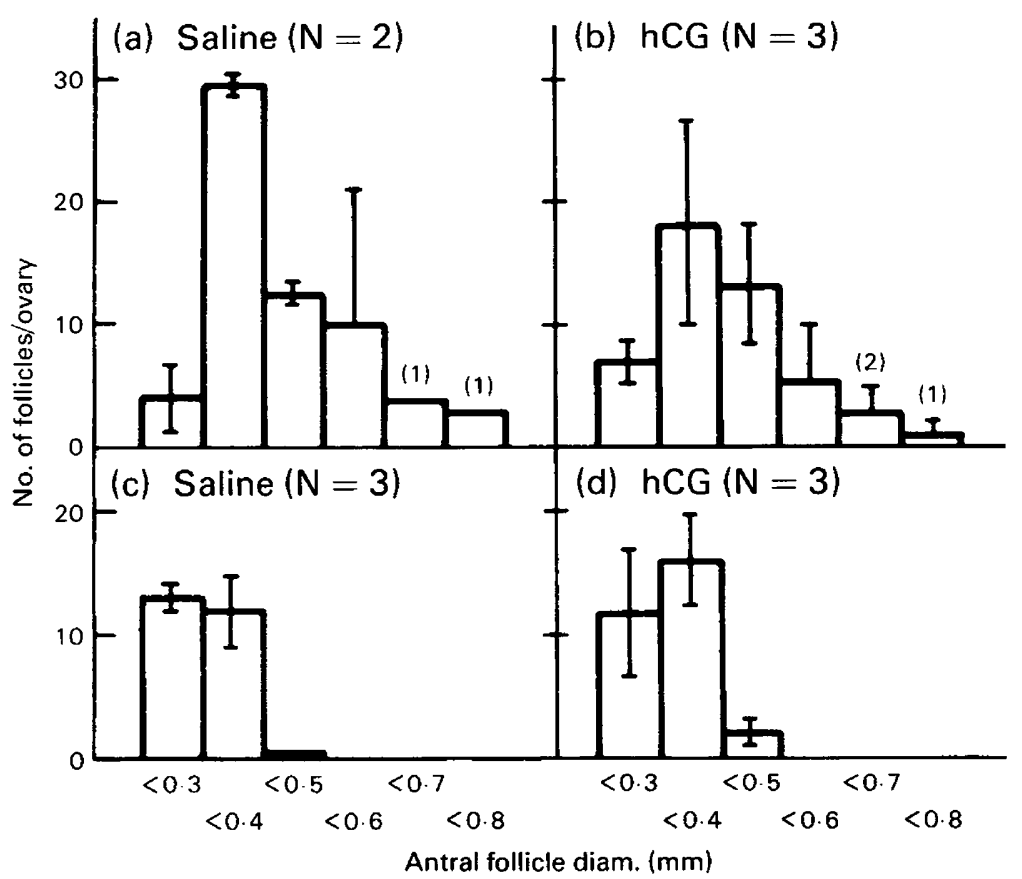

Text-fig. 3. Effects of saline and hCG treatment for $48 \mathrm{~h}$ on follicle size in 19-week-old ferrets housed in long days for 3 weeks $(a, b)$ or housed continuously in non-stimulatory short days $(c, d)$.

\section{Discussion}

The primary point to be made from these studies is that although one cannot distinguish females housed in a stimulatory photoperiod from their short-day controls on the basis of external appearance, body weight, or plasma oestradiol concentrations, distinct differences in ovarian morphology and physiology exist after 22 days of exposure to long days. The females receiving the puberty-inducing stimulus of long days exhibited both an increased incidence of large antral follicles and an increased ability to respond to an LH-like stimulus compared to the females still in short days. The ability to secrete oestradiol in response to an LH-like stimulus depends on two factors: (1) the presence of FSH-stimulated ovarian aromatase, and (2) LH receptors on the granulosa cells. The distribution of antral follicles in the ovaries did not markedly change with short-term ( $48 \mathrm{~h}$ ) hCG stimulation, suggesting that the exogenous hormone was acting on antral follicles already present in the ovary. LH receptors are induced by FSH, and hCG has very little FSH activity (Zeleznik, Midgley \& Reichert, 1974) which suggests that the sluggish response of short-day animals to the hCG stimulus could be due to relatively fewer LH receptors/cell than are found in females kept in long days. Alternatively, such an augmented response to hCG may reflect increased thecal testosterone production or increased granulosa cell aromatase enzymes in long-day females (Fortune \& Armstrong, 1977; Erickson \& Hsueh, 1978).

This evidence of ovarian stimulation after only 3 weeks of exposure to a stimulatory photoperiod is of interest when considering the mechanism of sexual maturation in ferrets in which there is increased frequency of $\mathrm{LH}$ pulsatile secretion and a marked decrease in negative feedback of oestradiol on LH secretion during photoperiod-induced sexual maturation (Ryan, 1984; Ryan \& Robinson, 1985). However, the elevation in pulsatile secretion of LH was not observed until 28-33 days after the onset of photostimulation, and so the ovarian development observed in females stimulated by long days in this study presumably occurred before the pubertal increase in LH secretion. 
FSH or prolactin or both hormones could be the mediators of the early ovarian stimulus induced by long days. However, studies of the adult ferret suggest that, if anything, FSH falls during the transition from anoestrus to oestrus (Donovan, Matson \& Kilpatrick, 1983), and we have not yet examined FSH patterns before puberty. A mechanism of a prolactin-mediated increase in ovarian response to $\mathrm{LH}$ has been proposed for the developing rat (Ojeda, Andrews, Advis \& Smith-White, 1980), but again, preliminary studies of prolactin secretion in adults suggest this will not be the case in ferrets: Smith, Forsyth \& Donovan (1983) failed to find any differences in basal prolactin concentrations between anoestrous and oestrous adult females housed in long days. Although such studies are suggestive, the initial pubertal ovarian stimulus provided by long days is unknown, and the potential involvement of FSH and/or prolactin must be examined in immature females because, at present, it is not known whether sexual maturation and the onset of seasonal breeding in adults occur by identical mechanisms.

I thank Mr Anthony F. Battelli and Mr Patrick T. Moran for help in developing and executing the surgical procedures and for excellent care and husbandry of the animals; Ms Susan Robinson for excellent handling of the technical aspects of the study including the radioimmunoassays; $\mathrm{Mr}$ Harold Schuler and the Department of Pathology at Magee-Womens Hospital for the histology on the ovaries; and Dr A. J. Zeleznik for helpful advice and discussion. This work was supported by grant HD 14489 from NICHHD.

\section{References}

Chang, M.C. (1950) Cleavage of unfertilized ova in immature ferrets. Anat. Rec. 108, 31-44.

Donovan, B.T. (1967) The effect of light upon reproductive mechanisms, as illustrated by the ferret. In Effects of External Stimuli on Reproduction (Ciba Fdn Symp. No. 26), pp. 43-52. Eds G. E. W. Wolstenholme \& M. O'Connor. Excerpta Medica, Amsterdam.

Donovan, B.T., Matson, C. \& Kilpatrick, M.J. (1983) Effect of exposure to long days on the secretion of oestradiol, oestrone, progesterone, testosterone, androstenedione, cortisol and follicle-stimulating hormone in intact and spayed ferrets. J. Endocr. 99, 361368 .

Erickson, G.F. \& Hsueh, A.J.W. (1978) Stimulation of aromatase activity by follicle stimulating hormone in vivo and in vitro. Endocrinology 102, 1275-1282.

Fortune, J.E. \& Armstrong, D.T. (1977) Androgen production by theca and granulosa isolated from proestrous rat follicles. Endocrinology 100, 13411347.

Hammond, J., Jr (1974) The ferret: some observations on photoperiod and gonadal activity, and their role in seasonal pelt and body weight changes; the synergistic effect of oestrogen and progesterone on weight gain; and a comparative study of the corpus luteum of the ferret and the rabbit. W. Heffer \& Son, Ltd, Cambridge.

Hammond, J. \& Marshall, F.H.A. (1930) Oestrus and pseudopregnancy in the ferret. Proc. R. Soc. B 105, 607-638.
Ojeda, S.R., Andrews, W.W., Advis, J.P. \& Smith-White, S. (1980) Recent advances in the endocrinology of puberty. Endocr. Rev. 1, 228-257.

Ryan, K.D. (1984) Hormonal correlates of photoperiodinduced puberty in a reflex ovulator, the female ferret (Mustela furo). Biol. Reprod. 31, 925-935.

Ryan, K.D. \& Robinson, S.L. (1985) A rise in tonic luteinizing hormone secretion occurs during photoperiod-stimulated sexual maturation of the female ferret. Endocrinology 116 (in press).

Scaramuzzi, J.R. \& Land, R.B. (1978) Oestradiol levels in sheep plasma during the oestrous cycle. J. Reprod. Fert. 53, 167-171.

Smith, S.V., Forsyth, I.A. \& Donovan, B.T. (1983) Study of prolactin levels in the ferret. J. Endocr. 99, 415421 .

Thorpe, D.H. (1967) Basic parameters in the reaction of ferrets to light. In Effects of External Stimuli on Reproduction (Ciba Fdn Symp. No. 26), pp. 53-66. Eds G. E. W. Wolstenholme \& M. O’Connor. Excerpta Medica, Amsterdam.

Zar, J.H. (1974) Biostatistical Analysis, ch. $11 \& 13$. Prentice-Hall Inc., Englewood Cliffs.

Zeleznik, A.J., Midgley, A.R., Jr \& Reichert, L.E., Jr (1974) Granulosa cell maturation in the rat: increased binding of human chorionic gonadotropin following treatment with follicle stimulating hormone in vivo. Endocrinology 95, 818-825.

Received 19 September 1984 